\title{
Pitfalls in conducting prospective trials in stage III cardiac amyloidosis - experience from the REVEAL study
}

\author{
EH Phillips², P Smith², L Clifton-Hadley², CJ Whelan'1, M Fontana'1, S Mahmood ${ }^{1}$, HJ Lachmann ${ }^{1}$, T \\ Adedayo $^{2}$, JD Gillmore ${ }^{1}$, PN Hawkins ${ }^{1}$ S Nash ${ }^{2}$, AD Wechalekar ${ }^{1}$, \\ National Amyloidosis Centre, Dept of Medicine, University College London, London ${ }^{1}$ \\ Cancer Research UK and University College London Cancer Trials Centre, London² \\ a.wechalekar@ucl.ac.uk
}

Very few prospective clinical trials have been conducted in advanced cardiac $A L$ amyloidosis $(A L)$ due to the rarity of the disease and challenging clinical course of the condition. With a median survival of only 7 months, cardiac AL represents a major area of unmet need (1). The introduction of proteasome inhibitors and immunomodulatory agents over the last decade has marked a new era in the treatment of plasma cell dyscrasias. The phase 1 CAN2007 study demonstrated the feasibility of single agent bortezomib in relapsed/refractory $A L$ up to a dose of $1.3 \mathrm{mg} / \mathrm{m}^{2}$ twice weekly (2). The REVEAL trial was conceived in 2008 to test the efficacy and toxicity of bortezomib-based triplet therapy in AL. Here, we report the course of the REVEAL trial, which illustrates the challenges in conducting trials where high mortality is part of the disease course.

REVEAL was designed as a parallel phase II trial assessing PAD (bortezomib, doxorubicin, dexamethasone) and CVD (cyclophosphamide, bortezomib, dexamethasone) for first line treatment of stage II-II cardiac AL. Subcutaneous bortezomib $1 \mathrm{mg} / \mathrm{m}^{2}$ and dexamethasone $20 \mathrm{mg}$ were given on days 1, 4, 8 and 11 of a 21-day cycle. Bortezomib could be increased to $1.3 \mathrm{mg} / \mathrm{m}^{2}$ if well tolerated and no evidence of early haematological response. Patients additionally received either weekly oral cyclophosphamide $350 \mathrm{mg} / \mathrm{m}^{2}$ (CVD arm; maximum dose 500mg) or intravenous doxorubicin $18 \mathrm{mg} / \mathrm{m}^{2}$ (PAD arm, day 1 only). Each arm was independent to allow for separate stopping rules. Patients with NYHA stage IV heart failure or significant left ventricular systolic dysfunction were excluded.

The study opened to recruitment in March 2012. Three early deaths occurred in the first 4 patients recruited (2 PAD, 1 CVD arm), all attributed to sudden cardiac death and within 11 weeks of study entry (see Table 1). The fourth patient (PAD arm) developed grade 4 corticosteroid-induced psychosis. All 3 deaths were assessed to be primarily amyloid-related. However in the interest of patient safety, recruitment was halted to allow data to be reviewed by the independent data monitoring committee (IDMC).

There was prolonged dialogue between the IDMC, sponsors and drug manufacturers. The trial reopened after 11 months with a redesign including mandatory safety reporting to the IDMC after every 3 patients. Ventricular arrhythmia was added as an exclusion criterion and patients were required to undergo 24-hour Holter monitoring prior to study entry. The chemotherapy protocol was changed to modified CVD (weekly bortezomib $1.3 \mathrm{mg} / \mathrm{m}^{2}$ and dexamethasone $20 \mathrm{mg}$ on days $1,8,15$ and 22 of a 35-day cycle with cyclophosphamide on days 1 and 15) or VD alone. One further early patient death occurred in the next 3 patients recruited (CVD arm). Although this was within predefined limits for 'expected outcome' for cardiac AL on this trial, as per protocol, recruitment was again suspended to allow data review by the IDMC.

Over the next 6 months, in discussion with the IDMC and sponsors, a number of further amendments were considered. At this juncture bortezomib was available for front line use and $>200$ patients had received upfront 
bortezomib (3), hence the impact on clinical practice of a small prospective study was felt to be limited. In light of published experience with use of CVD (4), we proposed closing the CVD arm and changing the VD arm to single agent escalating dose bortezomib. The ethics committee accepted these modifications but the UK regulatory authority (MHRA) deemed this a substantial amendment to trial design requiring full regulatory resubmission. The study was therefore formally closed in late 2014.

Baseline characteristics, treatment and responses for the 7 patients recruited are detailed in Table 1. Median age at enrolment was 57 years (range $34-68$ ). Three patients (43\%) had high risk stage Illb cardiac disease, defined by NT-proBNP $>8500 \mathrm{ng} / \mathrm{l}$ and/or systolic BP $<100 \mathrm{mmHg}(1)$, of which 2 died. The median number of treatment cycles delivered was $3(<1-6$ cycles). All 4 deaths $(57 \%)$ were attributed to sudden cardiac death/arrhythmia. Four patients $(57 \%)$ achieved at least very good partial haematological response within 1-3 cycles. The overall survival curve is shown in Figure 1. No relapses or deaths occurred in the 3 patients that completed treatment with 12.1 - 31.7 months follow-up.

Cardiac AL is known to have an early mortality of $23-42 \%(1,5)$. Given the small number of patients recruited to this study, it is unclear whether the early mortality was purely disease related or whether the drugs (bortezomib or any of the other agents) had an additional role. Of note, doxorubicin at a higher dose of $36 \mathrm{mg} / \mathrm{m}^{2}$ has been administered as part of both VAD in AL and PAD in myeloma without excess cardiotoxicity $(6,7)$.

It had been hoped that bortezomib might radically impact survival in cardiac AL, after the rapid responses seen in early studies. The experience from this study prompted detailed retrospective data review from UK, Italy and Germany resulting in 3 seminal publications comparing CDT with CVD (4), Mel-Dex with VMelDex (8) and a re-assessment of cardiac staging in AL amyloidosis, subdividing advanced cardiac AL into stage IIla and IIllb disease (1). Patients with stage IIlb disease are now recognised to have a median survival of 3 months (1). Chemotherapy has not been demonstrated to improve outcomes for the majority of these patients, apart from a very small proportion who obtain deep haematological remissions (3). Stage Illb cardiac AL is now considered a standard exclusion criteria in upfront AL trials (9).

A number of novel amyloid-specific treatments are in development, including drugs that accelerate amyloid fibril removal (10). It is now essential that prospective trials are rationally conducted in cardiac $\mathrm{AL}$ including patients at high risk of death. Building safeguards at each level is essential. However, this study showed that it is crucial for the IDMC, sponsors, regulators and funders to understand the complexity of AL. This will allow for understanding of the "expectedness" of events, including a very high incidence of early deaths, to avoid the pitfalls that led to prolonged delays at every stage and eventual closure of this trial. Although this study closed prematurely, it has provided valuable lessons, prompted retrospective studies that have immensely increased our understanding of $\mathrm{AL}$ and generated a new staging system and criteria which have now been adopted for trial entry.

Conflicts of interest: AW has received honoraria from Janssen-Cilag. All other authors report no conflicts of interest.

This trial was funded by Cancer Research UK (reference C23725/A11440) and registered at EudraCT (reference 2009-014906-33). Bortezomib was supplied free of charge by Janssen-Cilag. 


\section{References}

1. Wechalekar AD, Schonland SO, Kastritis E, Gillmore JD, Dimopoulos MA, Lane T, et al. A European collaborative study of treatment outcomes in 346 patients with cardiac stage III AL amyloidosis. Blood. 2013;121(17):3420-7. Epub 2013/03/13. doi: 10.1182/blood-2012-12-473066. PubMed PMID: 23479568.

2. Reece DE, Sanchorawala V, Hegenbart U, Merlini G, Palladini G, Fermand J-P, et al. Weekly and twiceweekly bortezomib in patients with systemic AL amyloidosis: results of a phase 1 dose-escalation study. Blood. 2009;114(8):1489-97. doi: 10.1182/blood-2009-02-203398.

3. Palladini G, Sachchithanantham S, Milani P, Gillmore J, Foli A, Lachmann H, et al. A European collaborative study of cyclophosphamide, bortezomib, and dexamethasone in upfront treatment of systemic AL amyloidosis. Blood. 2015;126(5):612-5. Epub 2015/05/20. doi: 10.1182/blood-2015-01-620302. PubMed PMID: 25987656.

4. Venner CP, Gillmore JD, Sachchithanantham S, Mahmood S, Lane T, Foard D, et al. A matched comparison of cyclophosphamide, bortezomib and dexamethasone (CVD) versus risk-adapted cyclophosphamide, thalidomide and dexamethasone (CTD) in AL amyloidosis. Leukemia. 2014;28(12):230410. doi: 10.1038/leu.2014.218.

5. Merlini G, Palladini G. Light chain amyloidosis: the heart of the problem. Haematologica. 2013;98(10):1492-5. Epub 2013/10/05. doi: 10.3324/haematol.2013.094482. PubMed PMID: 24091927; PubMed Central PMCID: PMCPmc3789451.

6. Mai EK, Bertsch U, Durig J, Kunz C, Haenel M, Blau IW, et al. Phase III trial of bortezomib, cyclophosphamide and dexamethasone (VCD) versus bortezomib, doxorubicin and dexamethasone (PAd) in newly diagnosed myeloma. Leukemia. 2015;29(8):1721-9. Epub 2015/03/20. doi: 10.1038/leu.2015.80. PubMed PMID: 25787915.

7. van Gameren II, Hazenberg BPC, Jager PL, Smit JW, Vellenga E. AL amyloidosis treated with induction chemotherapy with VAD followed by high dose melphalan and autologous stem cell transplantation. Amyloid. 2002;9(3):165-74. doi: 10.3109/13506120209114818.

8. Palladini G, Milani P, Foli A, Vidus Rosin M, Basset M, Lavatelli F, et al. Melphalan and dexamethasone with or without bortezomib in newly diagnosed AL amyloidosis: a matched case-control study on 174 patients. Leukemia. 2014;28(12):2311-6. doi: 10.1038/leu.2014.227.

9. Kastritis E, Leleu X, Arnulf B, Zamagni E, Cibeira MT, Kwok F, et al. A Randomized Phase III Trial of Melphalan and Dexamethasone (MDex) Versus Bortezomib, Melphalan and Dexamethasone (BMDex) for Untreated Patients with AL Amyloidosis. Blood. 2016;128(22):646-.

10.Richards DB, Cookson LM, Berges AC, Barton SV, Lane T, Ritter JM, et al. Therapeutic Clearance of Amyloid by Antibodies to Serum Amyloid P Component. New England Journal of Medicine. 2015;373(12):1106-14. doi: 10.1056/NEJMoa1504942. PubMed PMID: 26176329. 